\title{
Chromium Nucleosynthesis and Silicon-Carbon Shell Mergers in Massive Stars
}

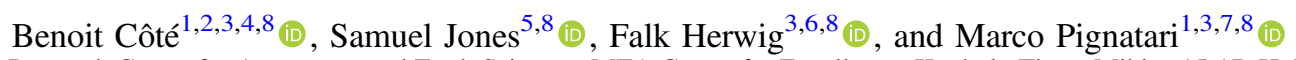 \\ ${ }^{1}$ Konkoly Observatory, Research Centre for Astronomy and Earth Sciences, MTA Centre for Excellence, Konkoly Thege Miklos 15-17, H-1121 Budapest, Hungary \\ benoit.cote@csfk.mta.hu \\ ${ }^{2}$ National Superconducting Cyclotron Laboratory, Michigan State University, East Lansing, MI 48824, USA \\ ${ }^{3}$ Joint Institute for Nuclear Astrophysics-Center for the Evolution of the Elements, USA \\ ${ }^{4}$ ELTE Eötvös Loránd University, Institute of Physics, Pázmány Péter sétány 1/A, H-1117 Budapest, Hungary \\ ${ }^{5}$ X Computational Physics (XCP) Division, Los Alamos National Laboratory, Los Alamos, NM 87545, USA \\ ${ }^{6}$ Department of Physics and Astronomy, University of Victoria, Victoria, Canada \\ ${ }^{7}$ E.A. Milne Centre for Astrophysics, Department of Physics \& Mathematics, University of Hull, Hull HU6 7RX, UK \\ Received 2019 June 11; revised 2020 February 3; accepted 2020 February 17; published 2020 March 27
}

\begin{abstract}
We analyze the production of the element $\mathrm{Cr}$ in galactic chemical evolution (GCE) models using the NuGrid nucleosynthesis yields set. We show that the unusually large $[\mathrm{Cr} / \mathrm{Fe}]$ abundance at $[\mathrm{Fe} / \mathrm{H}] \approx 0$ reported by previous studies using those yields and predicted by our Milky Way model originates from the merging of convective Si-burning and C-burning shells in a $20 M_{\odot}$ model at metallicity $Z=0.01$, about an hour before the star explodes. This merger mixes the incomplete burning material in the Si shell, including ${ }^{51} \mathrm{~V}$ and ${ }^{52} \mathrm{Cr}$, out to the edge of the carbon/oxygen (CO) core. The adopted supernova model ejects the outer $2 M_{\odot}$ of the $\mathrm{CO}$ core, which includes a significant fraction of the Cr-rich material. When including this $20 M_{\odot}$ model at $Z=0.01$ in the yields interpolation scheme of our GCE model for stars between 15 and $25 M_{\odot}$, we overestimate $[\mathrm{Cr} / \mathrm{Fe}$ ] by an order of magnitude at $[\mathrm{Fe} / \mathrm{H}] \approx 0$ relative to observations in the Galactic disk. This raises a number of questions regarding the occurrence of $\mathrm{Si}-\mathrm{C}$ shell mergers in nature, the accuracy of different simulation approaches, and the impact of such mergers on the presupernova structure and explosion dynamics. According to the conditions in this 1D stellar model, the substantial penetration of C-shell material into the Si shell could launch a convective-reactive global oscillation if a merger does take place. In any case, GCE provides stringent constraints on the outcome of this stellar evolution phase.
\end{abstract}

Unified Astronomy Thesaurus concepts: Galaxy chemical evolution (580); Chemical enrichment (225); Stellar abundances (1577); Massive stars (732); Chemical abundances (224); Stellar nucleosynthesis (1616); Galaxy abundances (574); Stellar evolutionary models (2046)

\section{Introduction}

Galactic chemical evolution (GCE) models and simulations are powerful tools to bridge nuclear astrophysics with astronomical observations (e.g., Tinsley 1980; Gibson et al. 2003; Nomoto et al. 2013; Matteucci 2014; Prantzos et al. 2018). Despite the complexity associated with simulating the formation and evolution of galaxies (e.g., Wise et al. 2012; Schaye et al. 2015; Somerville \& Davé 2015; Hopkins et al. 2018; Pillepich et al. 2018; Revaz \& Jablonka 2018), the fundamental input ingredients of all GCE studies are still the stellar yields (e.g., Romano et al. 2010; Mollá et al. 2015; Philcox et al. 2018). In the past years, we have developed an open-source GCE pipeline to bring nuclear astrophysics efforts to the forefront of GCE studies.

There are several sources of uncertainties in generating grids of stellar models for GCE applications, including, for example, uncertainties in nuclear reaction rates (e.g., Lugaro et al. 2004; Tur et al. 2009; Travaglio et al. 2014; deBoer et al. 2017; Nishimura et al. 2017; Denissenkov et al. 2018; Fields et al. 2018); stellar evolution and internal mixing (e.g., Meakin \& Arnett 2007; Sukhbold \& Woosley 2014; Jones et al. 2015; Davis et al. 2019); and supernova explosion modeling (e.g., Sukhbold et al. 2016; Fryer et al. 2018; Ebinger et al. 2019; Müller 2019; Couch et al. 2020). Turning this argument around, GCE studies are ideal framework to explore the impact of stellar processes in a broader astronomical context (Côté et al. 2017). In

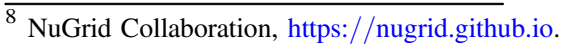

this study, we focus on the impact of shell mergers occurring in NuGrid massive star models (Ritter et al. 2018c) during the presupernova evolution phase (see also Rauscher et al. 2002; Mocák et al. 2018; Yadav et al. 2020).

Ritter et al. (2018a) have shown that oxygen-carbon (O-C) shell mergers could potentially be a relevant site for the production of odd- $Z$ elements and p-process isotopes at galactic scale. Andrassy et al. (2020), motivated by this, have studied the 3D hydrodynamical properties of $\mathrm{O}-\mathrm{C}$ shell mergers. Here, we discuss the impact of silicon-carbon ( $\mathrm{Si}-\mathrm{C})$ shell mergers on the evolution of chromium (Cr) in the Milky Way. Since the publication of the second set of NuGrid yields (Ritter et al. 2018c), an overproduction of $\mathrm{Cr}$ at galactic scale has been reported by Herwig et al. (2018) and Philcox et al. (2018) when using these yields in GCE codes. We have isolated the source of this overproduction. In the $20 M_{\odot}$ model at $Z=0.01$, a Si-C shell merger mixes large amounts of $\mathrm{Cr}$, synthesized during $\mathrm{Si}$ shell burning, above the assumed mass cut. ${ }^{9}$

In Section 2.1 we use our chemical evolution tools to highlight the $\mathrm{Cr}$ overproduction that points to the specific stellar model responsible for this overproduction. In the other subsections of Section 2, we present the relevant parts of the stellar model, show the implication of the $\mathrm{Si}-\mathrm{C}$ shell merger on the presupernova structure, and discuss the convective and burning timescales during the merger event. In Section 3, we conclude and raise open

\footnotetext{
9 Anything below the mass cut is locked inside the compact remnant and does not contribute to the ejected yields.
} 


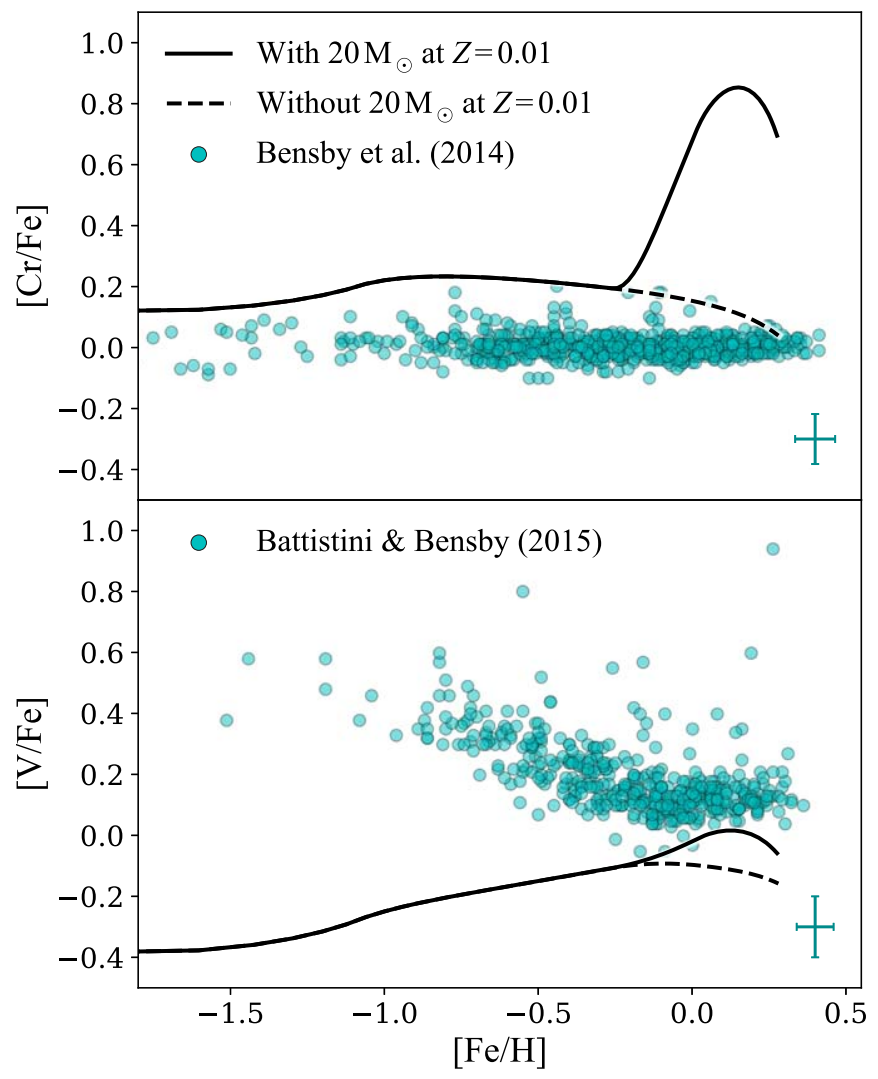

Figure 1. Predicted evolution of $[\mathrm{Cr} / \mathrm{Fe}]$ (top panel) and $[\mathrm{V} / \mathrm{Fe}]$ (bottom panel) as a function of $[\mathrm{Fe} / \mathrm{H}]$ for the Galactic disk using NuGrid yields (Ritter et al. 2018c) and the GCE code OMEGA+ (Côté et al. 2018). The solid and dashed lines show the predictions when including or excluding the $20 M_{\odot}$ model at $Z=0.01$, respectively. The cyan dots are stellar disk data from Bensby et al. (2014) and Battistini \& Bensby (2015).

questions regarding the occurrence of $\mathrm{Si}-\mathrm{C}$ shell mergers in nature and in multidimensional hydrodynamic simulations.

\section{Results}

\subsection{GCE Model}

We use the GCE code OMEGA+ (Côté et al. 2018) to bring NuGrid yields (Ritter et al. 2018c) into a galactic context. This code is part of the open-source JINAPyCEE python package ${ }^{10}$ and represents a one-zone GCE model surrounded by a large circumgalactic gas reservoir. The input parameters adopted in this study for regulating the star formation efficiency and the galactic inflow and outflow rates are available online. ${ }^{11}$ We use the initial mass function of Kroupa (2001). For SNe Ia, we use the yields of Iwamoto et al. (1999) and assume a total number of $10^{-3} \mathrm{SN}$ Ia per unit of stellar mass formed. As shown below, the overproduction of $\mathrm{Cr}$ is so strong that the choice of GCE parameters and SN Ia yields is of little importance.

The upper panel of Figure 1 shows the predicted evolution of $\mathrm{Cr}$ abundances as a function of $[\mathrm{Fe} / \mathrm{H}]$. Near $[\mathrm{Fe} / \mathrm{H}]=0$, our predictions (solid line) have a bump that overestimates disk data by almost an order of magnitude. The $20 M_{\odot}$ stellar model at $Z=0.01$ is at the origin of the Cr bump (see also Figure 7 in Herwig et al. 2018). When removing this stellar model from the

\footnotetext{
10 https://github.com/becot85/JINAPyCEE

11 https://github.com/becot85/JINAPyCEE/blob/master/DOC/OMEGA\% 2B_Milky_Way_model.ipynb
}

yields set, the bump disappears entirely (dashed line in Figure 1). The bottom panel shows a production of $\mathrm{V}$ accompanying the production of $\mathrm{Cr}$. This is not surprising, as $\mathrm{V}$ and $\mathrm{Cr}$ are made efficiently at similar stellar conditions (e.g., Woosley \& Weaver 1995). In agreement with the simulations reported here, $[\mathrm{V} / \mathrm{Fe}]$ is typically underestimated in GCE models at all metallicities compared with observations (e.g., Prantzos et al. 2018 and references therein).

\subsection{Final Evolutionary Stages of the Stellar Model}

Here we describe the evolution of the $20 M_{\odot}$ model at $Z=0.01$. We show that the source of $\mathrm{Cr}$ comes from the merging of the $\mathrm{C}$ - and Si-burning convective shells during the last hour of evolution before the model collapses. The Kippenhahn diagrams in Figure 2 show the evolution of convection zones (hatched regions) in the inner $6 M_{\odot}$ of the model. Convective boundary mixing was applied during the main sequence and the core He-burning phase, which likely contributed to the large CO and $\mathrm{Si}$ cores. No overshooting was applied after the He-core burning phase. We refer to Ritter et al. (2018c) for more details on this stellar model. At $t^{*} \equiv\left(t_{\text {collapse }}-t\right) / \mathrm{yr} \approx 10^{-3}$, there is a radiative layer that separates the convective Si-burning shell from the convective $\mathrm{C}$-burning shell above. At that time, as shown by the blue dashed line in Figure 3, the jump in entropy between the $\mathrm{Si}$ and $\mathrm{C}$ shells is relatively small compared with entropy barriers at the base of the $\mathrm{Si}$ shell (mass coordinate of $\sim 1.5 M_{\odot}$ ) and at the top of the $\mathrm{C}$ shell (mass coordinate of $\sim 5 M_{\odot}$ ).

Shortly after, as the $\mathrm{O}$ shell completely burns, the top of Si shell reaches the base of the $\mathrm{C}$ shell. From that time until $t^{*} \approx 10^{-4}$, the stratified $\mathrm{Si}^{-}$and C-burning convective shells share a convective boundary, which prevents the transport of material from the $\mathrm{C}$ shell into the $\mathrm{Si}$ shell, and vice versa. Finally, at $t^{*} \approx 10^{-4}$, the convective boundary is eroded and the Si-burning shell extends from the edge of the Fe core almost to the edge of the $\mathrm{CO}$ core. The top panel of Figure 2 shows that ${ }^{52} \mathrm{Cr}$ is mixed out to the edge of the $\mathrm{CO}$ core and its mass fraction is slightly reduced owing to dilution by the $\mathrm{C}$-shell material. The entropy profile at that time is shown by the orange solid line in Figure 3. The large entropy barriers at the base and the top of the Si-C shell prevent further mixing throughout the stellar interior.

As shown by our GCE model (Figure 1), a boost of [Cr/Fe] is generated above $[\mathrm{Fe} / \mathrm{H}] \approx-0.2$ when the yields of this $20 M_{\odot}$ model are included. One might expect that if the Siburning shell was mixed out and ejected, then $[\mathrm{Cr} / \mathrm{Fe}]$ should stay relatively flat, as both $\mathrm{Cr}$ and $\mathrm{Fe}$ are predominant products of Si burning. However, because the Si shell merges while it is still convective, and hence still burning, the ejected chemical signature is typical of incomplete Si burning.

$\mathrm{Si}$ burning produces the neutron-magic isotope ${ }^{52} \mathrm{Cr}$ relatively quickly, and thereafter produces ${ }^{56} \mathrm{Fe}$ via a sequence of capture and photodisintegration processes. This is illustrated in Figure 4, which shows the results of a one-zone nuclear reaction network calculation starting from pure ${ }^{28} \mathrm{Si}$ and evolved at density $\rho=2 \times 10^{6} \mathrm{~g} \mathrm{~cm}^{-3}$ and temperature $T=2.6 \mathrm{GK}$, which are approximately the conditions during shell $\mathrm{Si}$ burning in the $20 M_{\odot}$ stellar model about an hour before collapse. We note that the timescales of the burning will be different in the star, owing to the evolution of the core acting to increase the density and temperature of the shell. However, we have confirmed that the behavior is similar for a range of temperatures and densities. Only in the most extreme conditions $\left(10^{10} \mathrm{~g} \mathrm{~cm}^{-3}, 5 \mathrm{GK}\right)$ is $\mathrm{Cr}$ produced at the same time as Fe. 

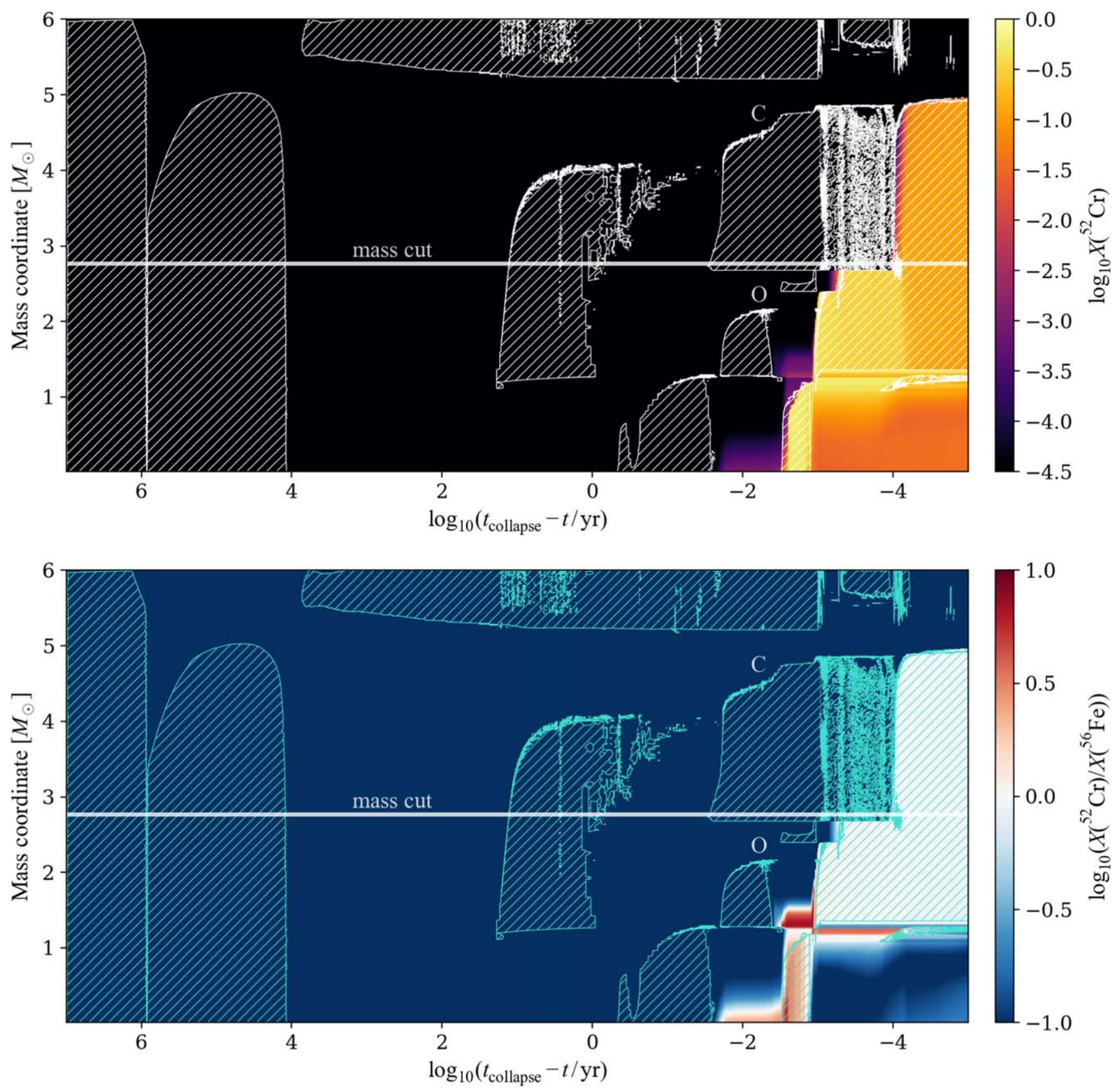

Figure 2. Kippenhahn (convective structure evolution) diagrams of the inner $6 M_{\odot}$ of the $20 M_{\odot}$ model at $Z=0.01$. The $x$-axis has an inverse logarithmic timescale showing the time remaining before collapse. Hatched contours show convectively unstable regions and color indicates the mass fraction of ${ }^{52} \mathrm{Cr}$ in the top panel and the ${ }^{52} \mathrm{Cr} /{ }^{56} \mathrm{Fe}$ ratio in the bottom panel. The ratio is approximately unity in the merged $\mathrm{C} / \mathrm{O} / \mathrm{Si}$ shell at the presupernova stage, which is the signature of incomplete $\mathrm{Si}$ burning that has been mixed throughout the merged shells. This is the predominant signature that appears in the ejected yields from the stellar model.

The core-collapse supernova (CCSN) explosion for this star was modeled by Ritter et al. (2018c) in the same way as described in Pignatari et al. (2016), with a mass cut at $2.77 M_{\odot}$ based on remnant mass prescription derived in Fryer et al. (2012). In this particular model with these assumptions, a significant fraction of the incomplete pre-SN Si-shell material, including ${ }^{52} \mathrm{Cr}$ and ${ }^{51} \mathrm{~V}$, is ejected during the explosion (the convective $\mathrm{Si}-\mathrm{C}$ shell extends up to $\sim 2 M_{\odot}$ above the assumed mass cut). The stellar yields used in our chemical evolution model account for the supernova shock and the explosive nucleosynthesis. The strong overproduction of $\mathrm{Cr}$ seen in Figure 1 shows that this pre-SN Sishell signature is not significantly destroyed during the explosion.

The overproduction of $\mathrm{Cr}$ and $\mathrm{V}$, relative to $\mathrm{Fe}$, during hydrostatic Si burning was already found by Thielemann \& Arnett (1985) and is not considered as an anomalous result. However, this material is typically buried in the compact remnant after the CCSN explosion, and therefore not ejected in the interstellar medium, or used as a seed for the explosive nucleosynthesis. Explosive Siburning material alone may carry a signature with a super-solar $\mathrm{Cr} / \mathrm{Fe}$ ratio (e.g., Woosley et al. 1973; Thielemann et al. 1996). A composition of explosive Si-burning and O-burning components in CCSN ejecta allowed to overcome such a problem but caused instead a general underproduction of $\mathrm{V}$ relative to $\mathrm{Fe}$ (e.g., Timmes et al. 1995; Goswami \& Prantzos 2000; Kobayashi et al. 2011).

\subsection{Supernova Implications}

The $2.77 M_{\odot}$ compact remnant is a black hole created by fallback (Fryer et al. 2012). In agreement with Sukhbold et al. (2016) and Ebinger et al. (2019), stars around $20 M_{\odot}$ are 


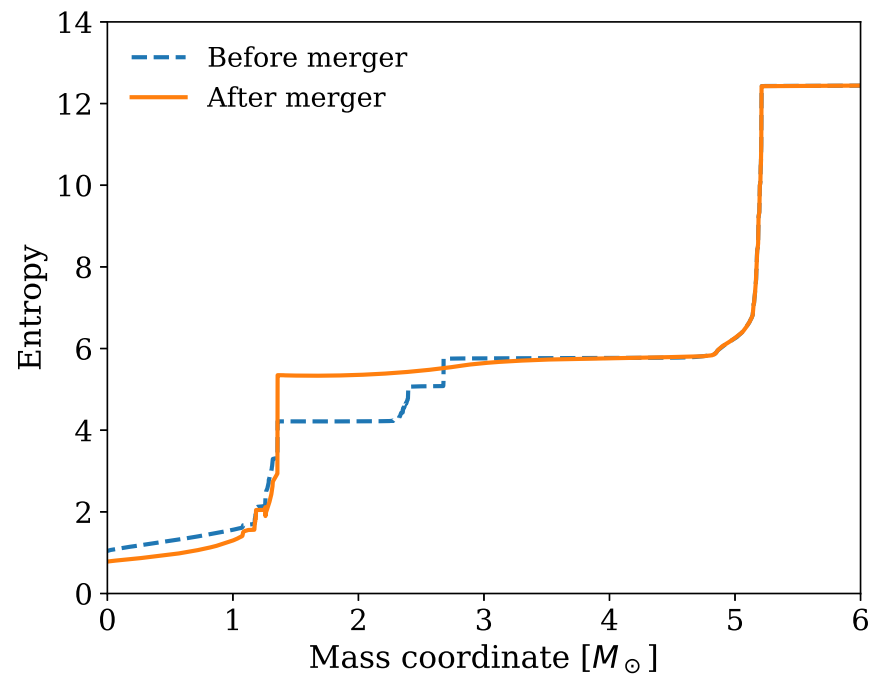

Figure 3. Entropy $\left[N_{\mathrm{A}} k_{\mathrm{B}}\right]$ in the core of the stellar model before and after the shell merger.

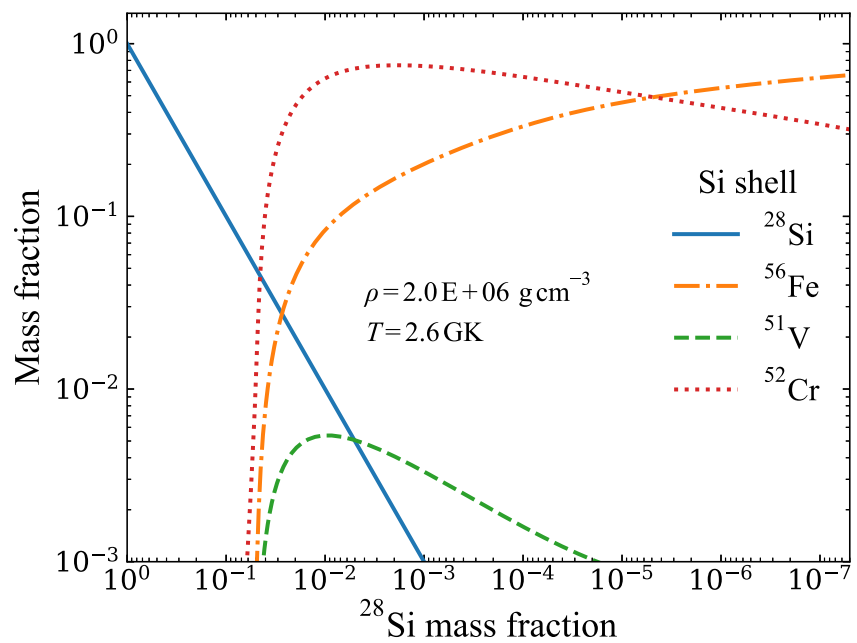

Figure 4. Evolution of key isotopes during a one-zone burn network integration at conditions characteristic of Si-shell burning in a $20 M_{\odot}$ star.

generally found to produce more failed explosions and black holes in 1D simulations than for lower initial masses.

Originally, the prescription of Fryer et al. (2012) was designed to reproduce the compact object mass distribution observed in the Milky Way, in particular the presence of a gap in the mass distribution between neutron stars and black holes (see Belczynski et al. 2012 and references therein). Given the nature of the prescription, the mass cut of the massive star models of Ritter et al. (2018c), including the $20 M_{\odot}$ model addressed in the present work, are set independently of the internal structure of the pre-SN model. This lack of connection between pre-SN structure and remnant mass adds uncertainties to whether or not the $\mathrm{Cr}$-rich material of this particular model should have been ejected at all. However, CCSN theory has far from settled on the precise relationship between progenitor structure and explosion properties (Sukhbold \& Woosley 2014; Müller 2016; Fryer et al. 2018). Even if we knew the remnant mass for a typical model of this initial mass and metallicity, the structure of the core in our model is affected by the shell merger in ways that are likely important in determining the dynamics of the collapse and subsequent explosion, or lack thereof, and compact remnant mass (e.g., Davis et al. 2019; Yadav et al. 2020).

Two of the most important properties of the progenitor models for determining the outcome of the CCSN are the stratification of density and electron fraction $\left(Y_{\mathrm{e}}\right)$. The left panel of Figure 5 shows how the $Y_{\mathrm{e}}$ profile is altered by the merger. The right panel shows that the shell merger erases the density jump that existed at the interface of the two shells (see arrow). Such a jump may facilitate the revival of the stalled CCSN shock wave owing to the rapid drop in accretion rate as the shell arrives at the shock radius (e.g., Buras et al. 2006; Ott et al. 2018). Although this particular density jump appears small, it could still be enough to alleviate the ram pressure at a critical time and allow for a successful explosion. Conversely, it may be that a more realistic simulation of this progenitor model results in direct black hole formation or formation of a much larger black hole than $2.77 M_{\odot}$ by fallback, in which case we would perhaps expect none of the $\mathrm{CO}$ core (and hence, none of the $\mathrm{Cr}$ ) to be ejected. Further implication for the SN explosion may derive from asymmetries that could be seeded right before the explosions in a shell merger, depending on the timescales for convection and burning during the merger.

\subsection{Convective Timescale}

The $Y_{\mathrm{e}}$ profile shown in Figure 5 raises another interesting point. The profile is not flat in the newly combined convection zone between 1.4 and $5 M_{\odot}$ (see solid orange line), as revealed by the time-dependent mixing, implemented in the diffusion approximation, when nuclear and mixing timescales are similar. The presupernova profile represents a state of incomplete mixing of the material in the two shells. The convective timescale $\tau_{\text {conv }}$, assuming it is approximately the time taken for a fluid element to complete one cycle of advection around a convective cell whose diameter is the shell's thickness, is given by

$$
\tau_{\text {conv }} \approx \frac{2 \pi r_{\text {cell }}}{\left\langle v_{\text {conv }}\right\rangle} \approx \frac{\left(7.4 \times 10^{9}\right) \pi \mathrm{cm}}{6.3 \times 10^{6} \mathrm{~cm} \mathrm{~s}^{-1}}=3690 \mathrm{~s} .
$$

The shell merger takes place $10^{-4} \mathrm{yr}$ or $3154 \mathrm{~s}$ before collapse, which is a similar timescale.

This raises the question of how efficient will be the mixing of ${ }^{52} \mathrm{Cr}$ and other Si-burning products into the outer core if there is only one turnover time to do it. Certainly the use of mixing length theory (MLT) for convection becomes inappropriate under these conditions because MLT only predicts convection properties in terms of averages over many convective turnover timescales.

\subsection{Burning Timescale during Shell Merger}

The constitution of the $\mathrm{C}$ shell should burn rapidly when exposed to the temperatures in the Si-burning shell. This energetic feedback will likely modify the flow dynamics and it should be considered when modeling the presupernova evolution of such a star (e.g., Herwig et al. 2014; Andrassy et al. 2020; Yadav et al. 2020).

We have estimated the burning timescale of ${ }^{12} \mathrm{C}$ by performing a simple nuclear network calculation beginning from $90 \%{ }^{28} \mathrm{Si}$ and $10 \%{ }^{12} \mathrm{C}$. We keep the temperature fixed at $3 \mathrm{GK}$ and the density at $1.4 \times 10^{8} \mathrm{~g} \mathrm{~cm}^{-3}$. We define the burning timescale as the e-folding time of the ${ }^{12} \mathrm{C}$ mass fraction 

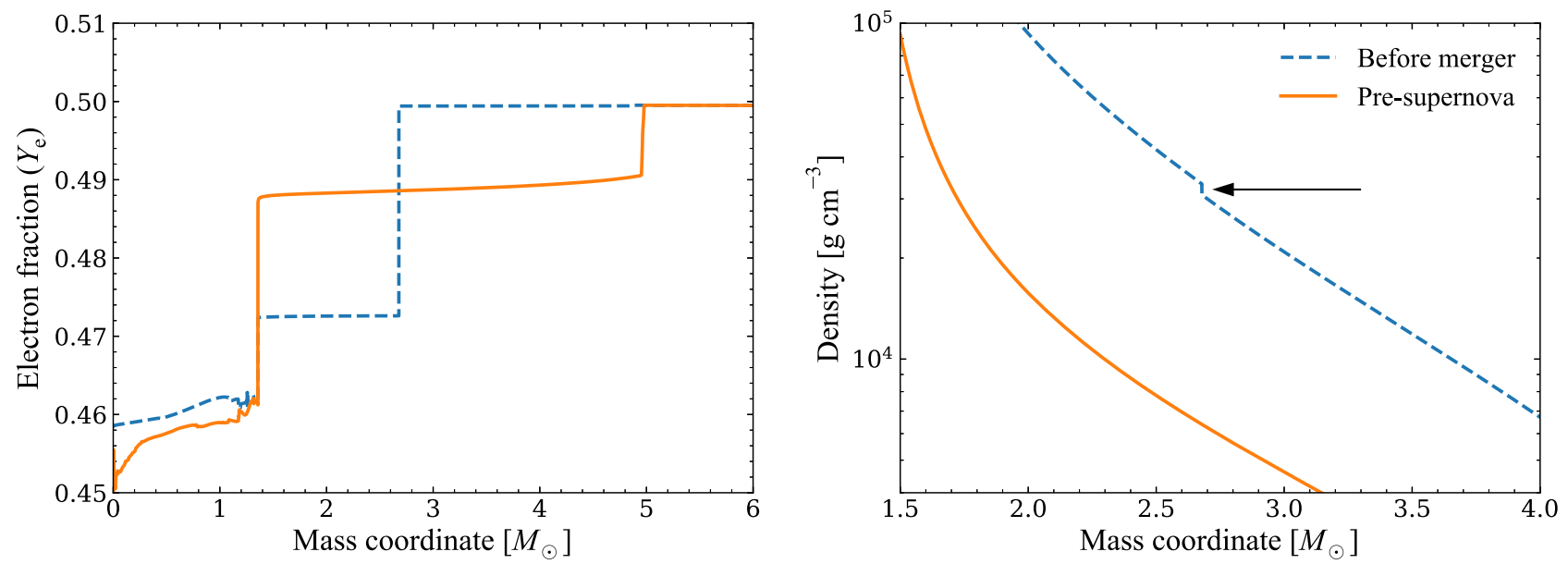

Figure 5. Electron fraction profile (left panel) and density profile (right panel) in the core of the stellar model before the shell merger and at the presupernova stage.

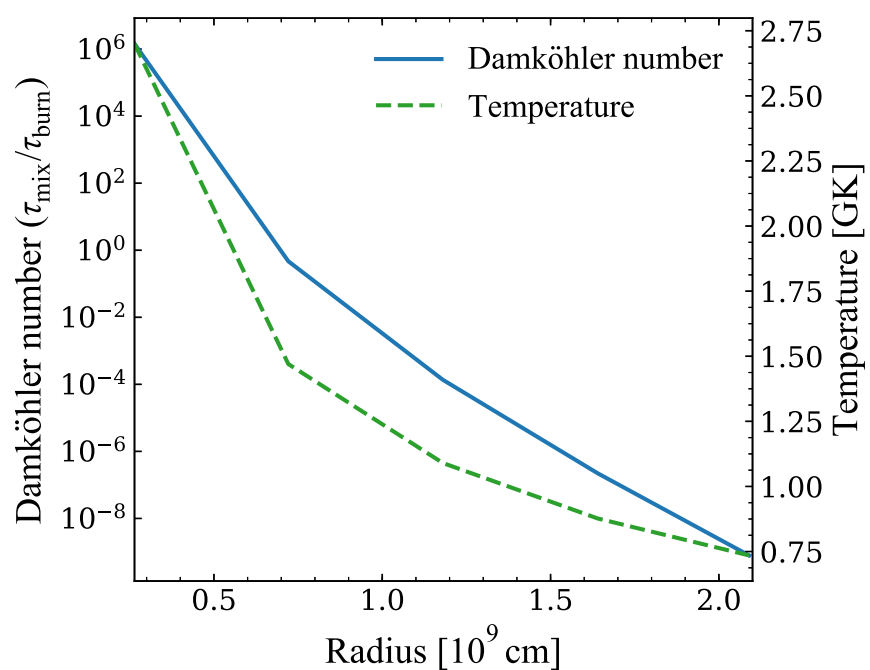

Figure 6. Damköhler number (blue solid line) and temperature (green dashed line) as a function of radius within the $\mathrm{Si}$ shell, about 20 cycles before the $\mathrm{Si}-\mathrm{C}$ shell merger. The range of radius shown represents the boundaries of the convective Si shell.

$X_{\mathrm{C}}$ under such conditions,

$$
\tau_{\text {burn }} \approx \frac{d t}{d \ln X_{\mathrm{C}}} .
$$

For example, near the bottom of the $\mathrm{Si}$ shell the timescale is $\sim 10^{-3} \mathrm{~s}$, which is much shorter than the $\sim 10^{3} \mathrm{~s}$ convective timescale, giving an exceptionally large Damköhler number of $\mathrm{Da}=\tau_{\text {mix }} / \tau_{\text {burn }} \approx 10^{6}$ (Figure 6). This means the material in the $\mathrm{C}$ shell will never actually reach the bottom of the convection zone. Instead, the situation is reminiscent of the $\mathrm{H}$-ingestion into He-shell convection in a post-AGB star. The distributed combustion flame is located where $\mathrm{Da} \sim 1$ (Herwig et al. 2011), which in this case is in the lower third of the $\mathrm{Si}$ convection zone. Depending on the energy release of the convective-reactive burn relative to the initial convective kinetic energy, a global non-spherical convective-reactive instability may ensue, such as the Global Oscillation of Shell H-ingestion (GOSH) that has been reported for $\mathrm{H}$-ingestion into He-shell flash convection (Herwig et al. 2014). The exact nature of the convective-reactive event and its impact on yields requires a more comprehensive 3D hydrodynamics and nucleosynthesis simulation approach which is beyond the scope of this work.

\section{Conclusions}

In this paper, we analyzed the $\mathrm{Si}-\mathrm{C}$ shell merger occurring in the $20 M_{\odot}$ model at $Z=0.01$ of the NuGrid collaboration (Ritter et al. 2018c). The $\mathrm{Si}-\mathrm{C}$ shell merger occurs roughly an hour before collapse. Following this event, a large amount of incomplete Si-burning material, including ${ }^{51} \mathrm{~V}$ and ${ }^{52} \mathrm{Cr}$, is mixed all the way from the $\mathrm{Si}$ core to a mass coordinate of $5 M_{\odot}$, which represents the upper boundary of the $\mathrm{C}$ shell as it was before the merger event. The convective timescale of this mixed shell is similar to the delay before the star collapses. As a firstorder approximation, it is therefore possible for the incomplete Si-burning material to mix and fill the $\mathrm{Si}-\mathrm{C}$ shell by the time of the explosion. Because the adopted mass cut for this model is $2.77 M_{\odot}$, a significant fraction of $\mathrm{Cr}$ is ejected during the explosion.

Using our GCE code OMEGA+ (Côté et al. 2018), and assuming that the ejecta of this specific $20 M_{\odot}$ model at $Z=0.01$ is representative of all $20 M_{\odot}$ stars at that metallicity, we overestimate the predicted evolution of $[\mathrm{Cr} / \mathrm{Fe}]$ in the Milky Way by almost an order of magnitude at $[\mathrm{Fe} / \mathrm{H}] \sim 0$. A question that emerges is whether or not $\mathrm{Si}-\mathrm{C}$ shell mergers occur in nature. From this experiment, the only conclusion we can draw from a GCE perspective is that the specific conditions (assumptions), in which this $20 M_{\odot}$ model evolves and explodes, cannot be representative of all $20 M_{\odot}$ stars and should be extremely rare if they occur at all. This type of event only occurred in one of the 20 NuGrid massive star models, but the exact probability for a massive star to experience a $\mathrm{Si}-\mathrm{C}$ shell merger cannot properly be quantified yet.

From the analysis of the stellar evolution model, the $\mathrm{Si}-\mathrm{C}$ merger could launch a non-spherical, global, convectivereactive instability similar to the GOSH found in H-ingestion in post-AGB stars (Herwig et al. 2014). Such an instability could seed substantial non-spherical perturbations of the initial conditions for the SN explosion. Another implication could be that such an instability enhances mixing of $\mathrm{Cr}$-rich Si-shell material into the $\mathrm{C}$ shell above. This would impact the amount of $\mathrm{Cr}$ ejected in this model. If such instabilities occur, their properties will depend on the detailed balance between energy produced from the entrainment of $\mathrm{C}$-shell material and the driving energetics of the Si-burning convection shell. Without 
such instability, if no or only a partial merger would take place, the convective mixing timescale in the $\mathrm{C}$ shell is similar to the remaining time to collapse, and dredge-up of $\mathrm{Cr}$ into the C-shell convection zone would likely be incomplete.

3D hydrodynamic simulations are required to investigate the range of mixing and burning conditions during interactions between the $\mathrm{Si}$ and $\mathrm{C}$ shells. Such an investigation would address important questions. How would a $\mathrm{Si}-\mathrm{C}$ shell merger look in multidimensional hydrodynamic simulations? To what extent would $\mathrm{Cr}$ make its way up into the $\mathrm{C}$ shell? How would an interaction between the $\mathrm{Si}$ and $\mathrm{C}$ shells impact the presupernova structure and the dynamics of the supernova explosion? Would the star explode? In any case, GCE would provide stringent constraints on the frequency and efficiency of $\mathrm{Cr}$ production through this process, which this study shows must remain small.

This research is supported by the ERC Consolidator Grant (Hungary) funding scheme (Project RADIOSTAR, G.A. n. 724560), by the National Science Foundation (NSF, USA) under grant No. PHY-1430152 (JINA Center for the Evolution of the Elements), and by the US Department of Energy LDRD program through the Los Alamos National Laboratory. Los Alamos National Laboratory is operated by Triad National Security, LLC, for the National Nuclear Security Administration of U.S. Department of Energy (Contract No. 89233218NCA000001). M.P. acknowledges significant support to NuGrid from STFC (through the University of Hull's Consolidated Grant ST/ R000840/1), and access to VIPER, the University of Hull High Performance Computing Facility. M.P. acknowledges the support from the "Lendulet-2014" Program of the Hungarian Academy of Sciences (Hungary). F.H. acknowledges funding through the NSERC Discovery Grant program. Part of this work has been carried out using the Compute Canada Arbutus cloud hosted by the University of Victoria.

Software: Jupyterlab (https://github.com/jupyterlab), JupyterHub (https://jupyterhub.readthedocs.io/en/latest), Cyberhubs Docker repository (https://hub.docker.com/u/cyberhubs), Docker (https://www.docker.com), Python (https://www. python.org), MESA (http://mesa.sourceforge.net), OpenMP (http://www.openmp.org), MESA-SDK (http://www.astro. wisc.edu/townsend/static.php?ref=mesasdk), MPI (https:// www.open-mpi.org), gfortran (https://gcc.gnu.org/fortran), SuperLU (http://crd-legacy.lbl.gov/xiaoye/SuperLU), OpenBLAS (http://www.openblas.net), OMEGA+ (Côté et al. 2018, SYGMA (Ritter et al. 2018b).

\section{ORCID iDs}

Benoit Côté (iD https://orcid.org/0000-0002-9986-8816 Samuel Jones (1D https://orcid.org/0000-0003-3970-1843 Falk Herwig (1) https://orcid.org/0000-0001-8087-9278 Marco Pignatari (i) https://orcid.org/0000-0002-9048-6010

\section{References}

Andrassy, R., Herwig, F., Woodward, P., \& Ritter, C. 2020, MNRAS, 491, 972 Battistini, C., \& Bensby, T. 2015, A\&A, 577, A9

Belczynski, K., Wiktorowicz, G., Fryer, C. L., Holz, D. E., \& Kalogera, V. 2012, ApJ, 757, 91
Bensby, T., Feltzing, S., \& Oey, M. S. 2014, A\&A, 562, A71

Buras, R., Janka, H. T., Rampp, M., \& Kifonidis, K. 2006, A\&A, 457, 281

Côté, B., O’Shea, B. W., Ritter, C., Herwig, F., \& Venn, K. A. 2017, ApJ, 835,128

Côté, B., Silvia, D. W., O'Shea, B. W., Smith, B., \& Wise, J. H. 2018, ApJ, 859,67

Couch, S. M., Warren, M. L., \& O'Connor, E. P. 2020, ApJ, 890, 127

Davis, A., Jones, S., \& Herwig, F. 2019, MNRAS, 484, 3921

deBoer, R. J., Görres, J., Wiescher, M., et al. 2017, RvMP, 89, 035007

Denissenkov, P., Perdikakis, G., Herwig, F., et al. 2018, JPhG, 45, 055203

Ebinger, K., Curtis, S., Fröhlich, C., et al. 2019, ApJ, 870, 1

Fields, C. E., Timmes, F. X., Farmer, R., et al. 2018, ApJS, 234, 19

Fryer, C. L., Andrews, S., Even, W., Heger, A., \& Safi-Harb, S. 2018, ApJ, 856,63

Fryer, C. L., Belczynski, K., Wiktorowicz, G., et al. 2012, ApJ, 749, 91

Gibson, B. K., Fenner, Y., Renda, A., Kawata, D., \& Lee, H.-c. 2003, PASA, 20,401

Goswami, A., \& Prantzos, N. 2000, A\&A, 359, 191

Herwig, F., Andrassy, R., Annau, N., et al. 2018, ApJS, 236, 2

Herwig, F., Pignatari, M., Woodward, P. R., et al. 2011, ApJ, 727, 89

Herwig, F., Woodward, P. R., Lin, P.-H., Knox, M., \& Fryer, C. 2014, ApJL, 792, L3

Hopkins, P. F., Wetzel, A., Kereš, D., et al. 2018, MNRAS, 480, 800 Iwamoto, K., Brachwitz, F., Nomoto, K., et al. 1999, ApJS, 125, 439

Jones, S., Hirschi, R., Pignatari, M., et al. 2015, MNRAS, 447, 3115

Kobayashi, C., Karakas, A. I., \& Umeda, H. 2011, MNRAS, 414, 3231

Kroupa, P. 2001, MNRAS, 322, 231

Lugaro, M., Ugalde, C., Karakas, A. I., et al. 2004, ApJ, 615, 934

Matteucci, F. 2014, The Origin of the Galaxy and Local Group, Saas-Fee Advanced Course, Vol. 37 (Berlin: Springer), 145

Meakin, C. A., \& Arnett, D. 2007, ApJ, 667, 448

Mocák, M., Meakin, C., Campbell, S. W., \& Arnett, W. D. 2018, MNRAS, 481, 2918

Mollá, M., Cavichia, O., Gavilán, M., \& Gibson, B. K. 2015, MNRAS, 451,3693

Müller, B. 2016, PASA, 33, e048

Müller, B. 2019, MNRAS, 487, 5304

Nishimura, N., Hirschi, R., Rauscher, T. St. J., Murphy, A., \& Cescutti, G. 2017, MNRAS, 469, 1752

Nomoto, K., Kobayashi, C., \& Tominaga, N. 2013, ARA\&A, 51, 457

Ott, C. D., Roberts, L. F., da Silva Schneider, A., et al. 2018, ApJL, 855, L3

Philcox, O., Rybizki, J., \& Gutcke, T. A. 2018, ApJ, 861, 40

Pignatari, M., Herwig, F., Hirschi, R., et al. 2016, ApJS, 225, 24

Pillepich, A., Springel, V., Nelson, D., et al. 2018, MNRAS, 473, 4077

Prantzos, N., Abia, C., Limongi, M., Chieffi, A., \& Cristallo, S. 2018, MNRAS, 476, 3432

Rauscher, T., Heger, A., Hoffman, R. D., \& Woosley, S. E. 2002, ApJ, 576, 323

Revaz, Y., \& Jablonka, P. 2018, A\&A, 616, A96

Ritter, C., Andrassy, R., Côté, B., et al. 2018a, MNRAS, 474, L1

Ritter, C., Côté, B., Herwig, F., Navarro, J. F., \& Fryer, C. L. 2018b, ApJS, 237, 42

Ritter, C., Herwig, F., Jones, S., et al. 2018c, MNRAS, 480, 538

Romano, D., Karakas, A. I., Tosi, M., \& Matteucci, F. 2010, A\&A, 522, A32

Schaye, J., Crain, R. A., Bower, R. G., et al. 2015, MNRAS, 446, 521

Somerville, R. S., \& Davé, R. 2015, ARA\&A, 53, 51

Sukhbold, T., Ertl, T., Woosley, S. E., Brown, J. M., \& Janka, H.-T. 2016, ApJ, 821,38

Sukhbold, T., \& Woosley, S. E. 2014, ApJ, 783, 10

Thielemann, F. K., \& Arnett, W. D. 1985, ApJ, 295, 604

Thielemann, F.-K., Nomoto, K., \& Hashimoto, M.-A. 1996, ApJ, 460, 408

Timmes, F. X., Woosley, S. E., \& Weaver, T. A. 1995, ApJS, 98, 617

Tinsley, B. M. 1980, FCPh, 5, 287

Travaglio, C., Gallino, R., Rauscher, T., et al. 2014, ApJ, 795, 141

Tur, C., Heger, A., \& Austin, S. M. 2009, ApJ, 702, 1068

Wise, J. H., Turk, M. J., Norman, M. L., \& Abel, T. 2012, ApJ, 745, 50

Woosley, S. E., Arnett, W. D., \& Clayton, D. D. 1973, ApJS, 26, 231

Woosley, S. E., \& Weaver, T. A. 1995, ApJS, 101, 181

Yadav, N., Müller, B., Janka, H. T., Melson, T., \& Heger, A. 2020, ApJ, 890, 94 